\title{
Microscopic hematuria and pelvic congestion syndrome in a patient with cirrhosis
}

Erich K Lang, Quan D Nguyen, Matthew H Smith, Karl Zhang

Departments of Radiology and Urology, SUNY Downstate Medical School, (EKL, QDN, MHS, KZ), NY and Department of Radiology, Johns Hopkins Medical Institutions (EKL), Baltimore

This 53 year old white female presented with complaints of severe pelvic congestion syndrome and hematuria. The patient gave a history of intermittent macro- and microscopic hematuria for the past 3 years. A recent urologic examination with blue light cystoscopy had revealed some submucosal varices in the bladder, which were thought to be the source of bleeding. However, 2 cystoscopies, 1 and 2 years prior, had not shown any varices. The patient had a history of ethanol abuse and at 2 occasions experienced hematemesis which was controlled by application of a Blackmore tube. A gynecologic examination complemented by ultrasound revealed a $3 \mathrm{~cm}$ submucosal fibroid. Laboratory finding were HB 12.8, HCT 38, RBC 4.2 mil, Platelets 180000, WBC 5800, BUN 28, Creatinine 1.3, estimate GFR 42, Na 136, K 5.6; $\mathrm{Cl} 98 \mathrm{mEq} / \mathrm{lt}$, alk pts $18 \mathrm{KA} \mathrm{U}$, Tot. Bil 0.6; Glucose 107, PT 10.0, INR 1.3, Transaminase 500U.

Urine-analysis revealed a spec grav 1022, $12 \mathrm{RBC} / \mathrm{hpf}, 1.2 \mathrm{WBC} / \mathrm{hpf}$, isolated clumps of bacteria; culture showed no growth. Physical examination noted pale complexion, distended abdomen with palotment suggesting the presence of ascitis; the spleen was markedly enlarged, BP 138/82, PR 78, Respiration 20 and regular.

An enhanced angioCT demonstrated massive esophageal and gastric fundus varices (Figure-1). The spleen was markedly enlarged. There were spleeno-renal shunts resulting in massive dilatation of the left renal vein (Figures 2,3). The left renal vein dilated to a degree, to be compromised in its passage between aorta and superior mesenteric artery (Figure-3). Dilated collateral veins to the umbilicus were present. In the pelvis one notes massive dilatation of parametrial veins; pelvic congestion syndrome (Figure 4).

Renal failure in cirrhotic patients is usually attributed to "hepato-renal syndrome" a consequence of albumen loss to ascitis. In our patients the massive spleno-renal shunt caused increased flow in the left renal vein and thereby increased left renal vein pressure (1). This was probably the cause for microscopic hemarturia that had been observed for 3 years (Figure-3). Moreover, the increased renal vein pressure caused parenchymal

Figure 1 - Demonstrates massive esophageal and juxta-esophageal collaterals in the retrocrural space extending into the posterior mediastinum.

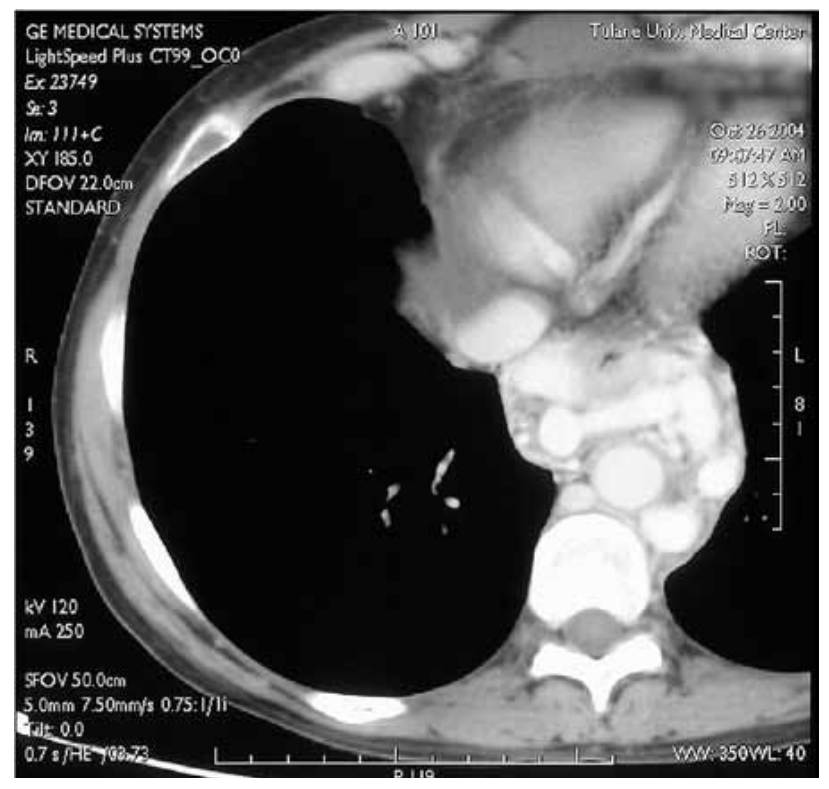


Figure 2 - Note the large spleen and prominent spleeno-renal shunts.

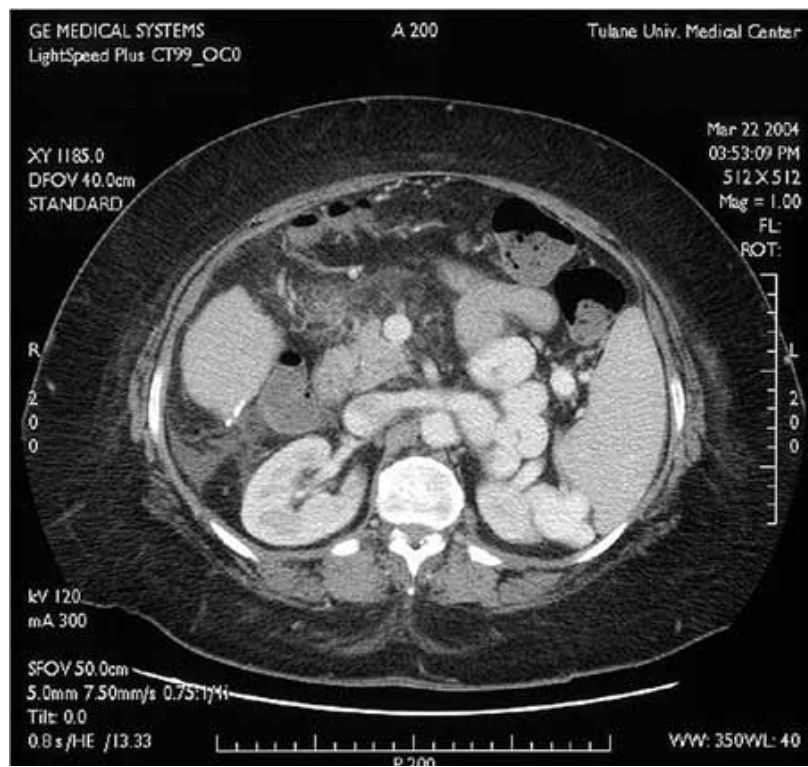

Figure 3 - The left renal vein is massively dilated and appears obstructed at its point of transgression between aorta and superior mesenteric artery; " nutcracker phenomenon”. The massive collateral flow has dilated the vein to a degree, to now cause obstruction. This in turn increases renal vein pressure and may cause microscopic hematuria.

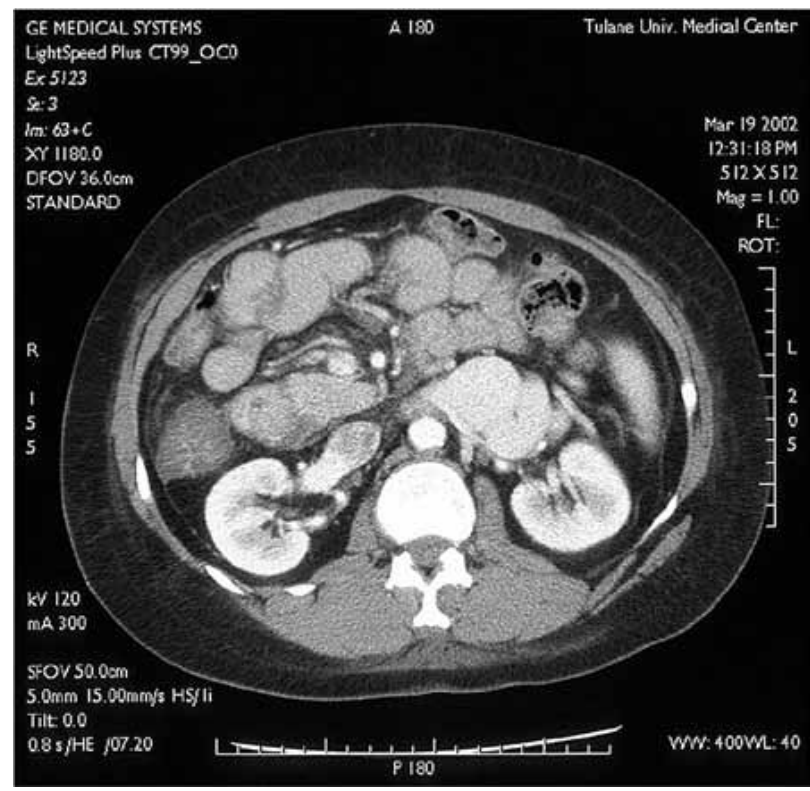

Figure 4 - Note the markedly dilated left parametrial vein, resulting from collateral flow from the gonadal vein. Collateral flow via pelvic veins is consistent with pelvic congestion syndrome.

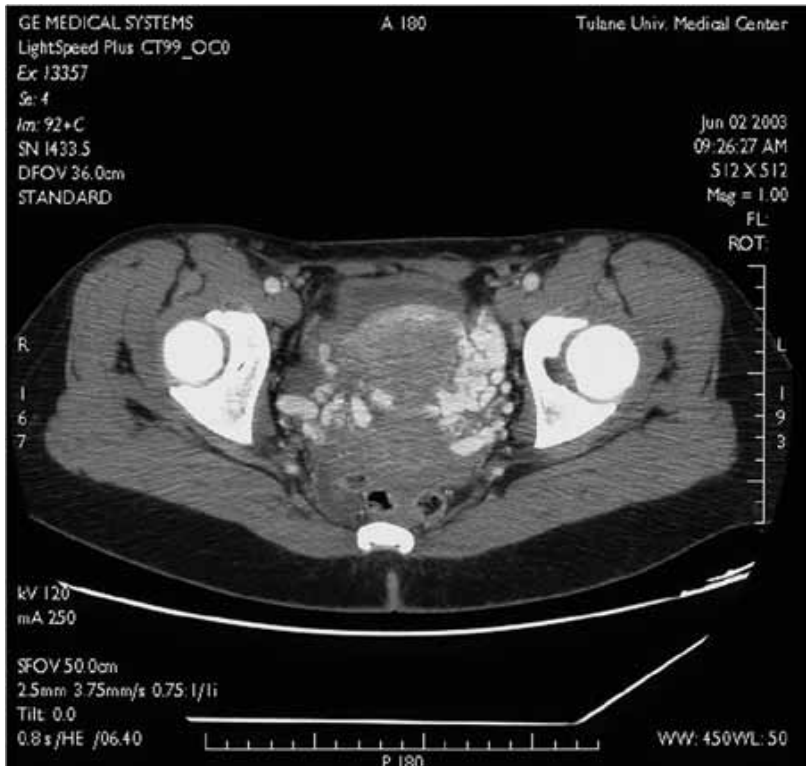

damage (2). Collateral decompression flow via the left gonadal vein occasioned pelvic congestion syndrome resulting in marked dilatation of the parametrial veins and collateral decompression flow via vesical veins. This in turn caused submucosal vesical varices, responsible for occurence of macroscopic hematuria.

\section{REFERENCES}

1. Tarantino G, Citro V, Conca P, Riccio A, Tarantino M, Capone $D$, et al.: What are the implications of the spontaneous spleno-renal shunts in liver cirrhosis? BMC Gastroenterol. 2009; 9: 89.

2. Gibo $\mathrm{M}$, Onitsuka $\mathrm{H}$ : Retroaortic left renal vein with renal vein hypertension causing hematuria. Clin Imaging. 1998; 22: 422-4.

Correspondence address: Dr. Erich K. Lang Departments of Urology and Radiology SUNY, Downstate Health Science Center 455 Lenox Road Brooklyn, NY, 11203, USA E-mail: erich.lang@downstate.edu 